\title{
Optical model with multiple band couplings using soft rotator structure
}

\author{
Dmitry Martyanov ${ }^{1, a}$, Efrem Soukhovitskii ${ }^{1}$, Roberto Capote ${ }^{2}$, Jose Manuel Quesada ${ }^{3}$, and Satoshi Chiba ${ }^{4}$ \\ 1 Nuclear Evaluation Lab, Joint Institute for Energy and Nuclear Research, Minsk, Belarus \\ 2 NAPC-Nuclear Data Section, International Atomic Energy Agency, Vienna, Austria \\ ${ }^{3}$ Departamento de Física Atómica, Molecular y Nuclear, Universidad de Sevilla, Sevilla, Spain \\ ${ }^{4}$ Laboratory for Advanced Nuclear Energy, Institute of Innovative Research, Tokyo Institute of Technology, Japan, and National \\ Astronomical Observatory of Japan, Tokyo, Japan
}

\begin{abstract}
A new dispersive coupled-channel optical model (DCCOM) is derived that describes nucleon scattering on ${ }^{238} \mathrm{U}$ and ${ }^{232} \mathrm{Th}$ targets using a soft-rotator-model (SRM) description of the collective levels of the target nucleus. SRM Hamiltonian parameters are adjusted to the observed collective levels of the target nucleus. SRM nuclear wave functions (mixed in $K$ quantum number) have been used to calculate coupling matrix elements of the generalized optical model. Five rotational bands are coupled: the ground-state band, $\beta-, \gamma-$, non-axial- bands, and a negative parity band. Such coupling scheme includes almost all levels below 1.2 MeV of excitation energy of targets. The "effective" deformations that define inter-band couplings are derived from SRM Hamiltonian parameters. Conservation of nuclear volume is enforced by introducing a monopolar deformed potential leading to additional couplings between rotational bands. The present DCCOM describes the total cross section differences between ${ }^{238} \mathrm{U}$ and ${ }^{232} \mathrm{Th}$ targets within experimental uncertainty from $50 \mathrm{keV}$ up to $200 \mathrm{MeV}$ of neutron incident energy. SRM couplings and volume conservation allow a precise calculation of the compound-nucleus $(\mathrm{CN})$ formation cross sections, which is significantly different from the one calculated with rigid-rotor potentials with any number of coupled levels.
\end{abstract}

\section{Coupled-channel optical model with soft-rotor couplings}

Tamura classical coupling model [1] has been recently extended to consider the coupling of collective rotational bands to the ground state band in even-even actinides including both axial and non-axial deformations [2-4]. These additional excitations were introduced as a perturbation to the underlying axial-symmetric rigid rotor structure of the ground state band (GSB) (i.e., $K$ was considered a good quantum number characterizing all collective bands). However, it is well known that the rigid-rotor calculated energy of the levels overestimates the measured excitation energy with increasing excitation energy. For ${ }^{238} \mathrm{U}$ nucleus, the $10^{+}\left(12^{+}\right)$state is $6 \%(8.4 \%)$ higher in energy than predicted by the rigid-rotor model.

A proper description of the energy of excited levels in actinides can be achieved by using a soft-rotator model (SRM) as demonstrated in Refs. [5,6]. SRM considers the stretching of the nucleus that lead to a higher momentum of inertia and lower excitation energies of high spin collective states in much better agreement with experimental data. Our goal is to use the SRM - reproducing the low-lying nuclear structure of even-even actinides with high accuracy - to predict coupling strengths and calculate corresponding matrix elements of the generalized dispersive optical model. The instant nuclear shape is described by small nonaxiality, and quadrupolar and octupolar departures

a e-mail: dmart@sosny. bas-net.by from the axially symmetric equilibrium shape as follows

$$
\begin{aligned}
R_{i}\left(\theta^{\prime}, \varphi^{\prime}\right)= & R_{i}^{r r}\left(\theta^{\prime}\right)+\delta R_{i}\left(\theta^{\prime}, \varphi^{\prime} ; \delta \beta_{2}, \gamma, \beta_{3}, \eta\right) \\
= & R_{0 i}\left\{1+\sum_{\lambda=2,4,6,8} \beta_{\lambda 0} Y_{\lambda 0}\left(\theta^{\prime}\right)\right\} \\
& +R_{0 i}\left\{\beta_{20}\left[\frac{\delta \beta_{2}}{\beta_{20}} \cos \gamma+\cos \gamma-1\right] Y_{20}\left(\theta^{\prime}\right)\right. \\
& +\left(\beta_{20}+\delta \beta_{2}\right) \frac{\sin \gamma}{\sqrt{2}}\left[Y_{22}\left(\theta^{\prime}, \varphi^{\prime}\right)+Y_{2-2}\left(\theta^{\prime}, \varphi^{\prime}\right)\right] \\
& +\beta_{3} \cos \eta Y_{30}\left(\theta^{\prime}\right)+\beta_{3} \frac{\sin \eta}{\sqrt{2}} \\
& \left.\times\left[Y_{32}\left(\theta^{\prime}, \varphi^{\prime}\right)+Y_{3-2}\left(\theta^{\prime}, \varphi^{\prime}\right)\right]\right\},
\end{aligned}
$$

where $R_{i}^{r r}$ is the rigid-rotor nuclear shape and $\delta R_{i}$ - the shape perturbations, $\theta^{\prime}$ and $\varphi^{\prime}$ are the angular coordinates in the body-fixed (intrinsic) system; $\beta_{\lambda 0}$ are the static axial deformations, $\beta_{2}=\beta_{20}+\delta \beta_{2}$ and $\beta_{3}$ are quadrupolar and octupolar deformations, and $\gamma$ and $\eta$ are the quadrupolar $(\gamma)$ and octupolar $(\eta)$ non-axial deformation parameters.

Incompressibility of the nuclear matter requires that nuclear deformations keep the nuclear volume constant. Eisenberg and Greiner introduced a dynamic monopolar term $R_{0 i} \beta_{00} Y_{00}$ [7] into the nuclear shape Eq. (1) to fulfil the constant volume condition. Considering $\delta \beta_{2}$ and $\beta_{3}$ small, and neglecting $\lambda>3$ deformations, we obtain (up to the second order of these parameters):

$$
\beta_{00}=-\left(2 \beta_{20} \delta \beta_{2}+\delta \beta_{2}^{2}+\beta_{3}^{2}\right) .
$$


Table 1. Soft-rotator nuclear Hamiltonian parameters and deformations for ${ }^{232} \mathrm{Th}$ and ${ }^{238} \mathrm{U}$ targets. Note that octupolar deformations are considered axially symmetric for these nuclei.

\begin{tabular}{lcc}
\hline Quantity & ${ }^{232} \mathrm{Th}$ & ${ }^{238} \mathrm{U}$ \\
\hline$\hbar \omega_{0}, \mathrm{MeV}$ & 0.702 & 0.979 \\
$\mu_{\beta}$ & 0.295 & 0.224 \\
$\mu_{\gamma}$ & 0.277 & 0.292 \\
$\gamma_{0}$ & 0.259 & 0.234 \\
$\mu_{\beta_{3}}$ & 0.030 & 0.062 \\
$B_{32}$ & 0.224 & 0.217 \\
$\eta$ & 0 & 0 \\
$\delta$ & 12.09 & 13.57 \\
$B_{42}$ & 0.035 & 0.00003 \\
$\beta_{20}$ & 0.201 & 0.221 \\
$\beta_{30}$ & 0.041 & 0.064 \\
$\beta_{4}$ & 0.067 & 0.056 \\
$\beta_{6}$ & -0.0074 & -0.0012 \\
\hline
\end{tabular}

Nonaxial $\gamma$ and $\eta$ deformation parameters do not change the volume thus they are not present in Eq. (2). Interband coupling strength is proportional to the "effective" deformations, which are the matrix elements over the collective coordinates of appropriate dynamic variables (details in Ref. [4]), while intraband coupling strength is the one determined in Ref. [1] with small correction terms arising from the nuclear softness [4] and volume conservation.

\section{Nuclear Hamiltonian parameters}

Comprehensive experimental data on low-lying collective levels are available for all long-lived or stable nuclei of practical interest. For even-even nuclei this allows determining nuclear Hamiltonian parameters using a soft rotator model of the nuclear structure [5,8]. SRM parameters determine the "effective "deformations needed for multiple-band coupling in the generalized optical model [4]. Our fitted SRM nuclear Hamiltonian parameters for ${ }^{238} \mathrm{U}$ and ${ }^{232} \mathrm{Th}$ targets are listed in Table 1 . The fitted SRM allows the assignment and description of almost all (about 25) experimentally observed levels with excitation energy below $1.2 \mathrm{MeV}$.

\section{Optical model parameters}

Above described modifications of the coupled-channel optical model have been implemented into the OPTMAN code $[8,9]$. The experimental data for nucleon interaction on ${ }^{232} \mathrm{Th}$ and ${ }^{238} \mathrm{U}$ targets coincide with the database used in our previous works [10,11]. A Lane consistent formulation of the generalized optical model [12] is used with dispersive integrals calculated analytically [13,14]. All scattering observables for neutron and proton induced reactions in the $0.001-200 \mathrm{MeV}$ range were employed in the potential search procedure. Fitted data included S0 and S1 strength functions, total cross section, angular distributions of scattered nucleons in $(n, n),(p, p)$ and $(p, n)$ reactions with excitation of isobaric analog states, and the ratio of ${ }^{238} \mathrm{U}$ and ${ }^{232} \mathrm{Th}$ total cross sections.

Derived Lane-consistent dispersive optical potential couples all the observed levels that belong to five rotational bands with excitation energies up to about $1.2 \mathrm{MeV}$ (GS: $I^{\pi}=0^{+} \ldots 10^{+}, \beta: I^{\pi}=0^{+} \ldots 6^{+}, \gamma: I^{\pi}=0^{+} \ldots 4^{+}$,

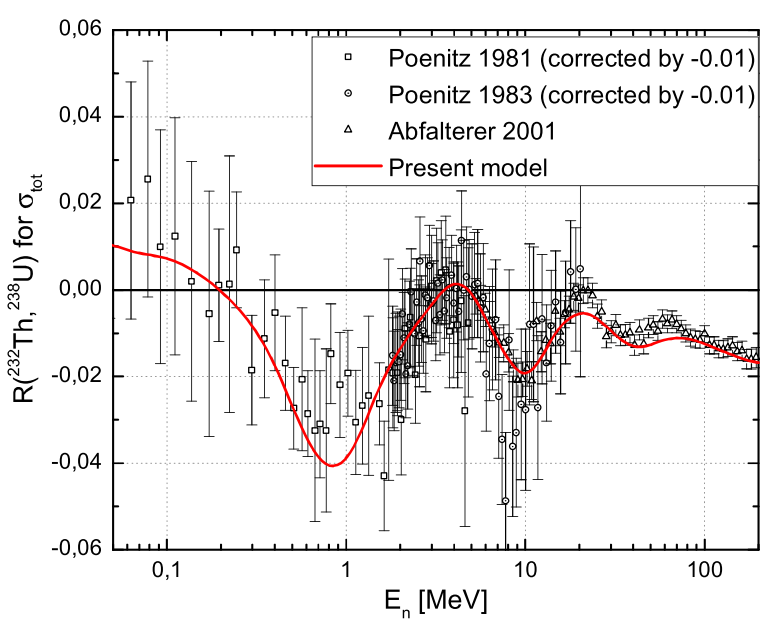

Figure 1. $R\left({ }^{238} \mathrm{U} ;{ }^{232} \mathrm{Th}\right)$ total cross section ratio defined as the total cross-section difference divided by the average total cross section: $R(A ; B)=\left(\sigma_{A}-\sigma_{B}\right) /\left(\frac{1}{2}\left(\sigma_{A}+\sigma_{B}\right)\right)$. Data from $[19,20]$.

NAX: $I^{\pi}=2^{+}, 3^{+}, 4^{+}$, OCT: $I^{\pi}=1^{-} \ldots 9^{-}$). There are 21 coupled levels plus the 3 lowest levels of the rotational band built on the isobar-analogue states - IAS. Table 2 lists the best-fit parameters of the present optical model (PM). The achieved overall $\chi^{2}=1.97$ implies an average model description of the experimental data within 1.5 times the experimental uncertainty for all observables.

A good agreement of calculated and experimental average resonance parameters can be seen in Table 3 . The calculated total neutron cross section data for ${ }^{232} \mathrm{Th}$ and ${ }^{238} \mathrm{U}$ targets were used to obtain the ratio $\mathrm{R}\left({ }^{232} \mathrm{Th},{ }^{238} \mathrm{U}\right)$ (defined as $\left.\mathrm{R}(\mathrm{A}, \mathrm{B})=\left(\sigma_{A}-\sigma_{B}\right) /\left(\frac{1}{2}\left(\sigma_{A}+\sigma_{B}\right)\right)\right)$. Description of measured $\mathrm{R}\left({ }^{232} \mathrm{Th},{ }^{238} \mathrm{U}\right)$ is recognized as a tough challenge for optical model potentials. The measured ratio derived from Poenitz [19] and Abfalterer [20] data has a small uncertainty, and displays an oscillatory behaviour with an amplitude from $-4 \%$ to about $+2 \%$ in the energy range from $50 \mathrm{keV}$ up to $200 \mathrm{MeV}$. An excellent agreement of PM with measured data is demonstrated in Fig. 1 for the whole energy range of measurements.

\section{Discussion}

What are the differences in calculated optical-model observables between the present model (PM) and previous works that used either SRM couplings $[5,6]$ or rigid-rotor model (RRM) couplings $[10,11]$ ? The main difference between SRM and RRM is the aforementioned coupling of the non-GS vibrational bands with strength proportional to the "effective" deformations derived from the SRM parameters. We have already coupled vibrational bands in Ref. [4], but we treated "effective" deformations for band coupling as additional fitting parameters. PM also accounts for nuclear volume conservation during shape oscillations and admixtures of states with different $K$ values in the nuclear wavefunctions (i.e., " $K$ mixing”).

Recently Dietrich et al. [21] showed that addition of $I^{\pi}=10^{+}$level to the GS-band $0^{+}, 2^{+}, 4^{+}, 6^{+}, 8^{+}$ coupling scheme within the RRM changes the compoundformation cross section $\sigma_{C N}$ by up to $4 \%$ for $n+{ }^{238} \mathrm{U}$ reaction at incident energies from $10 \mathrm{keV}$ up to about $2 \mathrm{MeV}$. Addition of the $12^{+}$level changed $\sigma_{C N}$ by only a few tenths of percent. Authors of Ref. [21] concluded that at 
Table 2. Dispersive coupled-channel optical model potential parameters for nucleon induced reaction on even-even actinides.

\begin{tabular}{|c|c|c|c|c|}
\hline & VOLUME & SURFACE & SPIN-ORBIT & COULOMB \\
\hline $\begin{array}{c}\text { Real } \\
\text { potential } \\
\text { parameters }\end{array}$ & $\begin{array}{l}V_{0}=50.92+ \\
\quad+0.0127(A-238) \mathrm{MeV} \\
\lambda_{H F}=0.00966 \mathrm{MeV}^{-1} \\
C_{\text {viso }}=20.28 \mathrm{MeV}\end{array}$ & - & $\begin{array}{l}V_{s o}=6.87 \mathrm{MeV} \\
\lambda_{s o}=0.005 \mathrm{MeV}^{-1}\end{array}$ & $C_{\text {Coul }}=1.0 \mathrm{MeV}$ \\
\hline $\begin{array}{c}\text { Imaginary } \\
\text { potential } \\
\text { parameters }\end{array}$ & $\begin{array}{l}A_{v}=11.51 \mathrm{MeV} \\
B_{v}=80.60 \mathrm{MeV} \\
E_{a}=54 \mathrm{MeV} \\
\alpha_{v}=0.355 \mathrm{MeV}^{1 / 2}\end{array}$ & $\begin{array}{l}W_{0}=16.37 \mathrm{MeV} \\
B_{s}=9.61 \mathrm{MeV} \\
C_{s}=0.01215 \mathrm{MeV}^{-1} \\
C_{\text {wiso }}=29.38 \mathrm{MeV}\end{array}$ & $\begin{array}{l}W_{s o}=-3.1 \mathrm{MeV} \\
B_{s o}=160 \mathrm{MeV}\end{array}$ & \\
\hline $\begin{array}{l}\text { Potential } \\
\text { geometry }\end{array}$ & $\begin{array}{l}r_{H F}=1.2547- \\
\quad-0.00117(A-238) \mathrm{fm} \\
a_{H F}=0.641+ \\
\quad+0.00182(A-238) \mathrm{fm} \\
r_{v}=1.2698 \mathrm{fm} \\
a_{v}=0.7000+ \\
\quad+0.00004(A-238) \mathrm{fm}\end{array}$ & $\begin{array}{l}r_{s}=1.1735+ \\
\quad+0.0048(A-238) \mathrm{fm} \\
a_{s}=0.618 \mathrm{fm}\end{array}$ & $\begin{array}{l}r_{s o}=1.1214 \mathrm{fm} \\
a_{s o}=0.59 \mathrm{fm}\end{array}$ & $\begin{array}{l}r_{c}=1.3934 \mathrm{fm} \\
a_{c}=0.610 \mathrm{fm}\end{array}$ \\
\hline
\end{tabular}

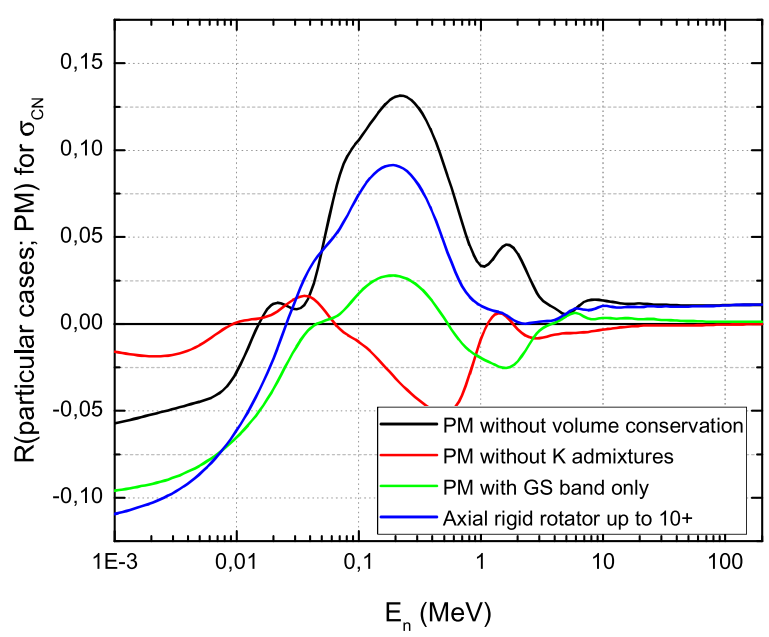

Figure 2. Dependence of the calculated $\sigma_{C N}(E)$ on different model assumptions plotted as a ratio to the $\sigma_{C N}(E)$ calculated with the full model potential (PM).

Table 3. Comparison of calculated and experimental average resonance parameters. The absolute uncertainties of evaluated strength functions are given in parentheses.

\begin{tabular}{|c|c|c|c|}
\hline Quantity & Reference & ${ }^{232} \mathrm{Th}$ & ${ }^{238} \mathrm{U}$ \\
\hline \multirow{4}{*}{$\begin{array}{l}S_{0} \times 10^{4} \\
(\mathrm{eV})^{-1 / 2}\end{array}$} & this work & 0.92 & 1.01 \\
\hline & RIPL-3 [15] & $0.84(.07)$ & $1.03(.08)$ \\
\hline & Porodzinski [16] & $0.80(.08)$ & $1.17(.10)$ \\
\hline & Mughabghab [17] & $0.71(.04)$ & $1.29(.13)$ \\
\hline \multirow{4}{*}{$\begin{array}{l}S_{1} \times 10^{4} \\
(\mathrm{eV})^{-1 / 2}\end{array}$} & this work & 1.75 & 1.71 \\
\hline & RIPL-3 [15] & $1.50(.30)$ & $1.60(.20)$ \\
\hline & Mughabghab [17] & $1.35(.04)$ & $2.17(.19)$ \\
\hline & CSEWG 1991 [18] & $1.60(.60)$ & $1.70(.30)$ \\
\hline \multirow{3}{*}{$R^{\prime}, \mathrm{fm}$} & this work & 9.68 & 9.55 \\
\hline & Mughabghab [17] & $9.65(.08)$ & $9.60(.10)$ \\
\hline & CSEWG 1991 [18] & $9.65(.30)$ & - \\
\hline
\end{tabular}

least 6 states must be coupled to yield stable results for $\sigma_{C N}$ over the entire energy range.

Calculated $\sigma_{C N}$ cross sections using variations of the PM (plotted as a ratio to PM results) are shown in Fig. 2. We have considered the impact on calculated cross sections of the volume conservation, $K$-mixing, the coupling scheme (full 21 levels vs ground-state band), and

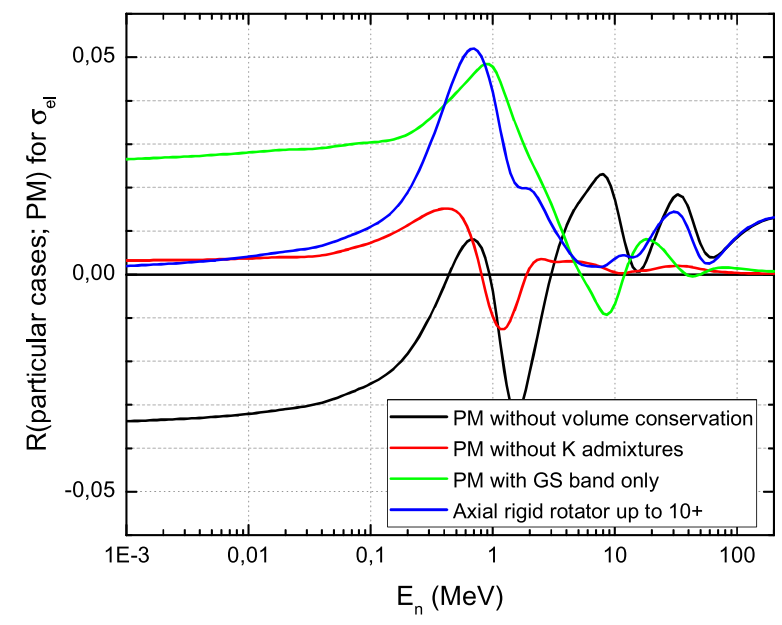

Figure 3. Dependence of the calculated $\sigma_{e l}(E)$ on different model assumptions plotted as a ratio to the $\sigma_{e l}(E)$ calculated with the full model potential (PM).

RRM vs SRM. Studied variations change the calculated $\sigma_{C N}$ from $-12 \%$ up to $+12 \%$, a large impact compared to what was observed by Dietrich et al. for the RRM.

For neutron incident energies below $3 \mathrm{MeV}$ reactionchannels couplings are much stronger. Hence, the discrepancies in calculated $\sigma_{C N}$ cross sections are larger. Two energy regions are clearly visible with a boundary around tens of $\mathrm{keV}$ : in the high energy region the models without account of volume conservation overestimate $\sigma_{C N}$, and viceversa for the lower energy region. The largest differences in Fig. 2 are seen for neglecting the volume conservation leading to a $-5 \% \sigma_{C N}$ at $1 \mathrm{keV}, \mathrm{a}+12 \%$ at $250 \mathrm{keV}$, and about $1 \%$ overestimation at energies above 7-8 MeV. The $\sigma_{C N}$ underestimation at higher energies can be easily understood as $\sigma_{C N}$ above $10 \mathrm{MeV}$ becomes closer to the geometric limit $\sigma_{C N} \sim R^{2}$, therefore the volume conservation condition effectively decreases the nuclear radius according to Eq. (2). Large differences in Fig. 2 are also seen between the PM and the axially symmetric rigidrotor model coupling levels up to $10^{+}$. Differences reach $-10 \%$ at $1 \mathrm{keV}$ and $\approx+9 \%$ at $200 \mathrm{keV}$. The non-axiality ( $K$-mixing) has smaller impact up to $-5 \%$.

A similar impact of model variations is shown in Fig. 3 for the calculated shape-elastic $\sigma_{\text {sel }}$ cross section on 
${ }^{238} \mathrm{U}$ target. Neglecting the nuclear volume conservation leads to shifts of the maxima in shape-elastic cross section, and thus to oscillations in the elastic cross section ratio above $100 \mathrm{keV}$ that can reach $3 \%$. Neglecting volume conservation below $100 \mathrm{keV}$ leads to an underestimation of the scattering radius $R^{\prime}$ by up to $3 \%$. $K$-mixing impact on elastic cross section is weak. However, the extended coupling scheme has again a large impact with differences between the PM with full coupling and RRM with coupled levels up to $10^{+}$reaching $+5 \%$ at $800-900 \mathrm{keV}$.

Therefore, the extension of the model couplings to vibrational levels in even-even actinides is an unavoidable condition for calculations of $\sigma_{e l}$ and $\sigma_{C N}$ cross sections with uncertainties below $10 \%$.

\section{Conclusion}

The number of model parameters in the optical model with multiple-band coupling [2-4] was reduced by using "effective" dynamic deformations from the soft rotator model [5] adjusted to reproduce the experimental collective levels scheme of the target nucleus. Therefore, the number of fitted parameters is equal to the number used in phenomenological rigid-rotor potentials.

A nuclear volume conservation condition was introduced to compensate volume variations due nuclear shape vibrations around a well-deformed axially symmetric equilibrium shape. The new model is implemented in coupled-channels optical model code OPTMAN. A dispersive optical potential parameters for ${ }^{232} \mathrm{Th}$ and ${ }^{238} \mathrm{U}$ nuclei were determined by least-square fitting with a $\chi^{2}=$ 1.97. 21 target levels from 5 rotational bands were coupled (+3 additional IAS levels for proton induced reactions).

Differences in calculated optical observables due to volume conservation may reach $10 \%$ and extend over the entire incident energy range from $1 \mathrm{keV}$ up to $20 \mathrm{MeV}$ and above. $K$-mixing in nuclear wave functions and vibrational-band couplings cannot be ignored if desired uncertainties are below $3 \%$. Differences of up to $10 \%$ in calculated $\mathrm{CN}$ formation cross section were observed if rigid-rotor model is used.

This work was partially funded by the Spanish Ministry of Economy and Competitivity under Contract
FPA2014-53290-C2-2-P. Presenting author (D.M.) is grateful to IAEA for financial support that allowed to attend the Conference.

\section{References}

[1] T. Tamura, Rev. Mod. Phys. 37, 679-708 (1965)

[2] J.M. Quesada, R. Capote, E.Sh. Soukhovitskiĩ and S. Chiba, Nucl. Data Sheets 118, 270-272 (2014)

[3] J.M. Quesada, R. Capote, E.Sh. Soukhovitskiĩ and S. Chiba, EPJ Web of Conf. 111, 03004 (2016)

[4] E.Sh. Soukhovitskĩ̃, R. Capote, J.M. Quesada, S. Chiba and D. Martyanov, submitted (2015)

[5] Yu.V. Porodzinskiĩ and E.Sh. Sukhovitskiĩ, Sov. J. Nucl. Phys. 53, 41-44 (1991)

[6] Yu.V. Porodzinkiĩ and E. Sukhovitskiĩ, Phys. At. Nuclei 59, 228-237 (1996)

[7] J.M. Eisenberg and W. Greiner, Nuclear Theory: Nuclear models, (North-Holland, Amsterdam, 1987)

[8] E.Sh. Soukhovitskiĩ et al., report JAERI-Data/Code 2005-002 (JAERI, Japan, 2005)

[9] E.Sh. Soukhovitskiĩ et al., report JAERI-Data/Code 2004-002 (JAERI, Japan, 2004)

[10] R. Capote, E.Sh. Soukhovitskiĩ, J. M. Quesada, and S. Chiba, Phys. Rev. C72, 064610 (2005)

[11] R. Capote et al., J. Nucl. Sc. Tech. 45, 333 (2008)

[12] J.M. Quesada, R. Capote, E.Sh. Soukhovitskiĩ, and S. Chiba, Phys. Rev. C76, 057602 (2007)

[13] J.M. Quesada, R. Capote, A. Molina, and M. Lozano, Comp. Phys. Commun. 153, 97 (2003)

[14] J.M. Quesada et al., Phys. Rev. C67, 067601 (2003)

[15] R. Capote et al., Nucl. Data Sheets 110, 3107 (2009)

[16] Yu.V. Porodzinskiĩ et al., report INDC(BLR)-12 (IAEA, Vienna, 1998)

[17] S.F. Mughabghab, Atlas of Neutron Resonances, $5^{\text {th }}$ edition, (Elsevier, Amsterdam, 2006)

[18] Cross Section Evaluation Working Group 1991. Technical report BNL-NCS-17541 (ENDF201), P.F. Rose (ed.), BNL (Upton, NY, USA, 1991)

[19] W.P. Poenitz et al., Nucl. Sci. \& Eng. 78, 333 (1981)

[20] W.P. Abfalterer et al., Phys. Rev. C63, 044608 (2001)

[21] F.S. Dietrich et al., Phys. Rev. C85, 044611 (2012) 